\title{
MARS: Über Sinn, Unsinn und Legalität eines umfassenden Gesundheitsinformationssystems
} Esther Krafta , Judith Wagner
Hanspeter Kuhn

a lic. rer. oec, stv. Leiterin Daten, Demographie und Qualität DDQ FMH

b Dr. sc. hum., Leiterin Medizinische Informatik und eHealth FMH

c Fürsprecher, Leiter Rechtsdienst, stv. Generalsekretär $\mathrm{FMH}$
Korrespondenz:

FMH

Abteilung Daten, Demographie und Qualität

Elfenstrasse 18

CH-3000 Bern 15

Tel. 0313591111

Fax 0313591112

esther.kraft[at]fmh.ch

\section{Einleitung}

Seit dem 1.1.2009 gilt im Krankenversicherungsgesetz (KVG) ein neuer Artikel; Art. 22a KVG sowie der dazugehörige Artikel in der Verordnung über die Krankenversicherung (KVV); Art. 31 KVV. Gemäss diesem Artikel sind die Leistungserbringer verpflichtet, «den zuständigen Bundesämtern Daten bekanntzugeben, die benötigt werden, um die Wirtschaftlichkeit und Qualität der Leistungen zu überwachen» [1]. Es sind unter anderem Angaben zur «Art der Tätigkeit», zur «Anzahl und Struktur der Patientinnen und Patienten in anonymisierter Form», $\mathrm{zu}$ «Art, Umfang und Kosten der erbrachten Leistungen» sowie zu «Qualitätsindikatoren» zu liefern [2]. Für die Erhebung und Sammlung der Daten ist das Bundesamt für Statistik (BFS) verantwortlich. Die Veröffentlichung der Ergebnisse, die gestützt auf Art. 22a KVG durch das BFS erhoben werden, erfolgt durch das Bundesamt für Gesundheit BAG.

$\mathrm{Zu}$ folgenden Themen kann das BAG Angaben oder Kennzahlen kommunizieren (KVV Art. 31):

- Leistungsangebot der Leistungserbringer

- Diplome und Weiterbildungstitel der Leistungserbringer

- Medizinische Qualitätsindikatoren

- Umfang und Art der erbrachten Leistungen

- Kostenentwicklungen.

Neben dem BAG werden die Daten weiteren Stellen zur Verfügung gestellt, wie z.B. dem eidgenössischen Preisüberwacher, dem Bundesamt für Justiz, den Kantonen, den Versicherern.

Anfang 2012 - gut drei Jahre nach Einführung des neuen KVG-Artikels - hat das BFS ein Grobkonzept einem breiten Kreis von Stakeholdern des Gesundheitswesens zur Verfügung gestellt. Das Projekt läuft unter dem Namen «Aufbau der Statistiken der ambulanten Gesundheitsversorgung MARS» [3].

\section{Zusammenfassung der Ziele und Inhalte des Projekts MARS}

Um die neuen KVG-Bestimmungen umzusetzen, plant das Bundesamt für Statistik den Aufbau der Statistiken der ambulanten Gesundheitsversorgung, der als ein «Beitrag für ein integrales statistisches Gesundheitsinformationssystem» verstanden werden soll. Mit diesem Informationssystem können laut BFS die «bestehenden gesetzlichen Vorgaben zur Dokumentation der Gesundheitsversorgung umgesetzt werden». Es wird also ein «Überblick über die Struktur der Leistungserbringer, die Diagnosen, die erbrachten Leistungen, die Inanspruchnahme der
Leistungen, die Behandlungspfade der Patienten, die Kosten sowie die Aus- und Weiterbildung des Personals» geschaffen. Gemäss Konzept wird die Bereitstellung von Grundlagen für die Versorgungsplanung, die epidemiologische Überwachung, die Wissenschaft sowie die Information der Öffentlichkeit angestrebt, um - ganz allgemein formuliert - die Entwicklungen des Gesundheitssystems verfolgen zu können. Das BFS gibt als Rechtsgrundlage für die Erhebung dieser Daten das Bundesstatistikgesetz (BstatG), die dazugehörige Verordnung sowie das Krankenversicherungsgesetz KVG Art. 21, Art. 22a und Art. 23 an.

Das vom BFS angestrebte Gesundheitsinformationssystem baut auf verschiedenen, je einen Themenbereich umfassenden Teilprojekten auf [3]:

- Teilprojekt 1: Strukturdaten Spital ambulant

- Teilprojekt 2: Patientendaten Spital ambulant

- Teilprojekt 3: Strukturdaten Arztpraxen

- Teilprojekt 4: Data-Warehouse

- Teilprojekt 5: Patientendaten Arztpraxen

- Teilprojekt 6: Patientendaten Spitex.

Bei der Art der Erhebung der Daten unterscheidet das BFS nicht zwischen administrativen und statistischen Zwecken. Für die Haltung und Verwendung der Daten sind jedoch unterschiedliche Methoden vorgesehen. Zu den statistischen Zwecken gehören beispielweise die epidemiologische Beschreibung und Analyse des Gesundheitszustandes der Bevölkerung, Datenlieferungen an internationale Organisationen und Information der Öffentlichkeit über das Gesundheitswesen. Die administrativen Daten sollen Vollzugsaufgaben ermöglichen, wie z.B. die Umsetzung des Krankenversicherungsgesetztes oder Planungs- und Steuerungsaufgaben. Die Daten zu den verschiedenen Teilprojekten werden teilweise mit schon bestehenden Erhebungen erfasst, teilweise sind die Erhebungen neu zu schaffen. Im Hinblick auf das vom BFS geplante integrale Gesundheitsinformationssystem sieht das Konzept insbesondere die Verknüpfung der Patientendaten explizit vor.

\section{Position der FMH zum geplanten Aufbau der Statistik}

Die FMH ist von der Notwendigkeit von verlässlichen Zahlengrundlagen überzeugt und setzt sich dafür ein, dass das Schweizerische Gesundheitssystem über diese verfügt. Sie unterstützt daher eine methodisch korrekte und die gesetzlichen Grundlagen respektierende Erhebung von Kennzahlen zur stationären und ambulanten Gesundheitsversor- 
gung und in der Folge auch die Möglichkeit zur Schliessung allfälliger Lücken bzgl. internationaler oder europäischer Erhebungen. Einige zentrale Inhalte des Konzepts MARS entsprechen jedoch aus Sicht der FMH diesen Anforderungen in keiner Weise. Die Kritik bezieht sich insbesondere auf folgende Punkte des Projekts:

\section{Gesetzestexte aus dem Krankenversicherungsgesetz KVG}

\section{Art. 22a Daten der Leistungserbringer}

1 Die Leistungserbringer sind verpflichtet, den zuständigen Bundesbehörden die Daten bekannt zu geben, die benötigt werden, um die Anwendung der Bestimmungen dieses Gesetzes über die Wirtschaftlichkeit und Qualität der Leistungen zu überwachen. Namentlich sind folgende Angaben zu machen:

a. Art der Tätigkeit, Einrichtung und Ausstattung, Rechtsform;

b. Anzahl und Struktur der Beschäftigten und der Ausbildungsplätze;

c. Anzahl und Struktur der Patientinnen und Patienten in anonymisierter Form;

d. Art, Umfang und Kosten der erbrachten Leistungen;

e. Aufwand, Ertrag und finanzielles Betriebsergebnis;

f. medizinische Qualitätsindikatoren.

${ }^{2}$ Die befragten natürlichen und juristischen Personen sind zur Auskunft verpflichtet. Die Angaben sind kostenlos zur Verfügung zu stellen.

${ }^{3}$ Die Angaben werden vom Bundesamt für Statistik erhoben. Es stellt die Angaben nach Absatz 1 zur Durchführung dieses Gesetzes dem Bundesamt für Gesundheit, dem Eidgenössischen Preisüberwacher, dem Bundesamt für Justiz, den Kantonen und Versicherern sowie den in Artikel 84a aufgeführten Organen je Leistungserbringer zur Verfügung. Die Daten werden veröffentlicht. ${ }^{4}$ Der Bundesrat erlässt nähere Vorschriften zur Erhebung, Bearbeitung, Weitergabe und Veröffentlichung der Daten unter Wahrung des Verhältnismässigkeitsprinzips.

\section{Art. 23 Statistiken}

${ }^{1}$ Das Bundesamt für Statistik erarbeitet die notwendigen statistischen Grundlagen zur Beurteilung von Funktions- und Wirkungsweise dieses Gesetzes. Es erhebt zu diesem Zweck bei den Versicherern, den Leistungserbringern und der Bevölkerung die notwendigen Daten.

${ }^{2}$ Die befragten natürlichen und juristischen Personen sind zur Auskunft verpflichtet. Die Informationen sind kostenlos zur Verfügung zu stellen.

${ }^{3}$ Das Bearbeiten von Daten zu statistischen Zwecken erfolgt nach dem Bundesstatistikgesetz vom 9. Oktober 1992.

\section{Verordnung über die Krankenversicherung KVV}

\section{Art. 31 Veröffentlichung der Daten der Leistungserbringer}

1 Das BAG veröffentlicht die Ergebnisse der vom Bundesamt für Statistik gestützt auf Artikel 22a des Gesetzes und durch das BAG nach Artikel 51 des Bundesgesetzes vom 26. Juni $2006^{2}$ über die universitären Medizinalberufe erhobenen Daten so, dass namentlich folgende Angaben oder Kennzahlen der sozialen Krankenversicherung nach Leistungserbringer oder nach Kategorien von Leistungserbringern ersichtlich sind:

a. Leistungsangebot der Leistungserbringer;

b. Diplome und Weiterbildungstitel der Leistungserbringer;

c. medizinische Qualitätsindikatoren;

d. Umfang und Art der erbrachten Leistungen;

e. Kostenentwicklung.
- Gesundheitslebenslauf: Die FMH lehnt die geplante Erstellung eines Gesundheitsinformationssystems $a b$, für das keine gesetzliche Grundlage besteht: Man würde damit faktisch über einen «Gesundheitslebenslauf» eines jeden Bürgers verfügen, ohne dass sich die Schweizer Bevölkerung je aufgrund eines referendumsfähigen Bundesgesetzes zur Frage äussern konnte, ob sie dies will.

- Erhebung über alle Patienten: Das BFS will die Ärzte im Rahmen der Statistik dazu verpflichten, sämtliche Patientenkontakte bis und mit Selbstzahler den Behörden zu melden. Auch dafür besteht aus Sicht der FMH keine gesetzliche Grundlage. Die meldenden Ärzte sehen sich der Gefahr ausgesetzt, dass sie gegen das Berufsgeheimnis verstossen und sich somit strafbar machen.

- Vollerhebung: Die geplante Vollerhebung über jeden ambulanten Behandlungsfall ist aus Sicht der FMH in keiner Weise sachlich und fachlich begründet. Sie würde die Ärzte mit zusätzlichen administrativen Aufgaben massiv belasten und damit auch den Zielen des aktuellen Masterplans für die Hausarztmedizin diametral widersprechen. Das BFS rechnet nach eigenen Angaben mit 1,5 Mio. Datensätzen pro Jahr. Es würde vor allem bei den Leistungserbringern, die diese Daten erfassen müssen, ein sehr hoher Aufwand entstehen, aber auch bei den verarbeitenden kantonalen und Bundesstellen. Diese Kosten müssten letztlich die Steuer- und Prämienzahler begleichen. Jedoch konnte der konkrete Nutzen der Erhebung bisher nicht aufgezeigt werden.

Keine wirksame Anonymisierung: Das beschriebene Vorgehen garantiert in keiner Weise eine Anonymisierung der Patientendaten. Art. 22a KVG nennt Angaben über «Anzahl und Struktur der Patientinnen und Patienten in anonymisierter Form» und garantiert dem Bürger im Zusammenhang mit der Statistik - nota bene im Unterschied zur früheren Fassung von Art. $30 \mathrm{KVV}$ ausdrücklich Anonymität [4]. Die Leistungserbringer dürfen aufgrund des klaren Wortlauts keine personenbezogenen Einzeldaten liefern, die Rückschlüsse auf die Personen zulassen oder gar mit einem eindeutigen Identifikator versehen sind [5]. $\mathrm{Zu}$ melden sind aufgrund von Art. 22a KVG beispielsweise «20 COPD-Fälle». Unzulässig sind hingegen Meldungen wie «bei Patient X wurde COPD diagnostiziert, zudem leidet er an Diabetes, ist alkoholsüchtig und inkontinent.»

- Die Zielsetzung und Fragestellungen sind für die Konzeptionierung einer solchen Erhebung viel zu unpräzise formuliert. Das Krankenversicherungsgesetz verlangt Daten, um die «Wirtschaftlichkeit und Qualität der Leistungen zu überwachen» [1] - nicht mehr, aber auch nicht weniger. Auf diese konkrete, im Gesetz festgehaltene Zielformulierung wird im Konzept zu wenig ein- 
gegangen. Am Beispiel der Erhebung der Qualitätsindikatoren wird klar ersichtlich, dass ein Konzept fehlt, welches aufzeigt, wie man mit den erhobenen Daten die Zielsetzungen und Fragestellungen erreichen könnte (Proof of concept). Im Übrigen sind für die Bestimmung der Qualitätsindikatoren nicht alle gemäss Konzept zu erhebenden Daten erforderlich.

\section{Schlussfolgerungen}

Die FMH ist überzeugt, dass das Schweizer Gesundheitswesen verlässliche und methodisch korrekt erhobene Daten benötigt, sei es beispielsweise für die Sicherstellung der medizinischen Versorgung der Schweizer Bevölkerung oder für die Planung der Ausund Weiterbildung der Ärzteschaft. Die Datensammlung darf jedoch nicht ohne klaren Auftrag des Gesetzgebers die Schaffung eines Gesundheitslebenslaufs von der Wiege bis zur Bahre ermöglichen, dem der Patient nur durch Behandlung im Ausland entgehen könnte. Damit würde das Arztbzw. Patientengeheimnis - sogar für Selbstzahler! faktisch aufgehoben. Dies darf nicht geschehen. Die
FMH setzt sich im Dialog mit den zuständigen Stellen für eine Gesundheitsstatistik ein, die die nötigen Planungsgrundlagen sicherstellt und gleichzeitig die Anonymität der Patienten zuverlässig garantiert.

\section{Literatur}

1 Art. 22a Abs.1 KVG.

2 Kraft E. Art. 22a KVG: Fluch oder Segen; Schweiz Ärztezeitung. 2010;91(36):1367-9.

3 MARS: Modules Ambulatoires des Relevés sur la Santé, Version 1.0, Juni 2012.

4 «Diese [Angaben] sollen ein Bild über die Tätigkeit der Leistungserbringer ermöglichen, indem sowohl die Infrastruktur bzw. die Aus- und Weiterbildung wie auch die erbrachten Leistungen und die dabei entstehenden Kosten anzugeben sind, ohne dabei die Anonymität der Patientinnen und Patienten in Frage zu stellen.» Botschaft KVG-Revision Spitalfinanzierung, BBl. 2004. S. 5573.

5 Wenn nur genügend Daten gesammelt werden, ist es auch bei pseudonymisierten Daten einfach, die betroffene Person herauszufinden. Deshalb sieht Art. 22a KVG zu Recht nur Angaben über die Patientenstruktur und nicht über einzelne Patienten vor.

\section{Durchblick bei Rechtsfragen.} Der Leitfaden für juristisches Basiswissen.

«Rechtliche Grundlagen im medizinischen Alltag». Für Mitglieder kostenlos.

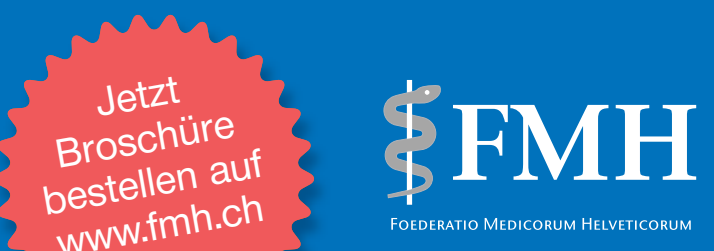

